\title{
Re-Embedding the Disembedded
}

\author{
Die Umweltstandards multinationaler Konzerne in der globalen Steuerungsar- \\ chitektur $^{1}$
}

\section{Von Martin Herberg}

\section{Globaler Rechtspluralismus, private Regulative und das Konzept der Interlega- lität}

Der Prozess der Globalisierung führt zu zahlreichen Verschiebungen im Gleichgewicht der gesellschaftlichen Kräfte und Mächte; darunter auch zu einer Verlagerung öffentlicher Funktionen von der Staaten- in die Wirtschaftswelt. Während es dem Nationalstaat immer weniger gelingt, die Handlungszusammenhänge im transnationalen Raum wirksam unter Kontrolle zu bringen, lassen sich zahlreiche Initiativen nicht-staatlicher Akteure beobachten, um die Lücken der institutionellen Ordnung auszufüllen. Multinationale Unternehmen, Versicherungen, Banken und Anbieter von Internet-Dienstleistungen, sie alle sind mit der Erarbeitung und Durchsetzung eigener, problemfeldspezifischer Standards befasst; als globale, nichtstaatliche Normgebungsagenturen wirken sie mit an der Entstehung von länderübergreifenden Ordnungsstrukturen außerrechtlichen Charakters, die gleichwohl zahlreiche Merkmale mit staatlich gesetztem Recht teilen. »Es ist dem Recht weder begriffswesentlich, dass es vom Staat ausgehe, noch auch, dass es die Grundlage für die Entscheidungen der Gerichte oder anderer Behörden [...] abgebe« (Ehrlich 1989, S.17) - so die These aus der Gründerzeit der Rechtssoziologie, die durch die Emergenz eines informellen Rechts der Weltgesellschaft neue Brisanz erhält (vgl. Günther/ Randeria 2000; Teubner 2000).

$\mathrm{Zu}$ den Merkmalen dieser quasi-rechtlichen Strukturen gehört, dass sie aus mehreren, eng verzahnten Normen und Regeln auf unterschiedlichen Ebenen bestehen, die konkret genug sind, um klare Grenzlinien zwischen zulässigen und unzulässigen Praktiken zu etablieren. Häufig kann die Einhaltung der Regeln nicht einfach der freiwilligen Folgebereitschaft der Beteiligten überlassen werden, was die Einrichtung spezialisierter Überwachungs- und Durchsetzungsorgane zur Folge hat (zur Bedeutung solcher Spezialorgane für den Begriff des Rechts vgl. Luhmann 1987, S.79). Hinzu kommt das Merkmal der normativen Verankerung der Steuerungsarrangements in einer Schicht tiefer liegender Geltungsgrundlagen; teils resultiert die Geltung der Regeln aus den krisenhaften Erfahrungen der Vergangenheit und der Nicht-Hintergehbarkeit der erfolgten Lerneffekte; teils ist es die Befolgung der Regeln über längere Zeiträume, der diese ihre Bestandskraft verdanken, und teilweise wird die Verbindlichkeit auch dadurch gesteigert, dass die Übernahme der betreffenden Aufgaben mit einer öffentlichen Selbstverpflichtung verknüpft wird, wodurch als weiterer Garant die eigene Glaubwürdigkeit ins Spiel gebracht wird.

Für die soziologische Globalisierungsforschung, die politische Theorie internationaler Beziehungen und die juristische Diskussion über die Zukunft des Rechts stellen solche Regelungen privater Provenienz ein ergiebiges, gleichzeitig aber auch prekäres Untersuchungsfeld dar; prekär und schwer handhabbar sind die emergenten Ordnungsstrukturen zum einen

1) Der Aufsatz dokumentiert einige Ergebnisse aus dem Überschneidungsbereich zweier Forschungsprojekte; dem abgeschlossenen DFG-Projekt »Doppelstandards bei Auslandsinvestitionen« (Forschungsstelle für Europäisches Umweltrecht Bremen) und dem laufenden Teilprojekt »Transnational Governance« im Rahmen des Sonderforschungsbereichs 597 »Staatlichkeit im Wandel«. Wertvolle Anregungen verdanke ich Prof. Ulrich Oevermann, Prof. Eva Senghaas-Knobloch, Prof. Gerd Winter sowie den TeilnehmerInnen der Tagung »Wirtschaft der Gesellschaft« in Siegen 7.-8. April 2005.

Soziale Welt 56 (2005), S. 399 - 416 
deshalb, weil der Forscher permanent mit den etatistischen Bürden seiner eigenen Disziplin konfrontiert wird - sei es der nationalstaatlich geprägte Gesellschaftsbegriff der modernen Soziologie (kritisch: Beck 1997, S.48ff) oder der rechtspositivistische Bias im juristischen Diskurs (vgl. Lampe 1995) -, prekär sind sie aber auch aufgrund ihrer geringen Transparenz, der fehlenden oder nur schwach ausgebildeten Beteiligungs- und Mitsprachemöglichkeiten Dritter und der problematischen Rückbindung an die Normen des geltenden staatlichen und zwischenstaatlichen Rechts - »the attribution of public functions to private actors directly challenges democratic and liberal theories of governance and law « (Cutler 2002, S.33). Für viele Autoren signalisiert die aktuelle Entwicklung eine Ablösung staatlicher Handlungsfähigkeit durch private Steuerung; ganze Politikfelder werden, so die Befürchtung, staatlichem Einfluss entzogen und von privaten Akteuren »usurpiert (Pauly 2002, S.76), während der Staat sich zunehmend in einer passiven und marginalisierten Rolle wieder findet.

Schlagwortartig auf den Punkt gebracht werden diese Bedenken in der Rede von der Entstehung einer neuen »Niemandsherrschaft « (Beck 2002, S.102). Die Tatsache, dass viele relevante Entscheidungen hinter verschlossenen Türen gefällt werden, die Unübersichtlichkeit der Zuständigkeiten und die fehlende Publizität der geltenden Standards - von manchen Autoren als Defizit in der Dimension der accountability, der Zurechenbarkeit und Nachprüfbarkeit politisch relevanter Entscheidungen thematisiert (Bovens 1998) - sind für die gesellschaftlichen Akteure vis á vis der Wirtschaftswelt mit einem gesteigerten Maß an Unsicherheit, einer Schwächung ihrer Einflussmöglichkeiten und somit, jedenfalls bis zu einem gewissen Grad, mit einer Beschneidung ihrer Bürgerrechte verbunden - auch wenn die quasi-rechtlichen Normsysteme in manch anderer Hinsicht die Funktion erfüllen, diese Rechte zu schützen. Diese eigentümliche Zweischneidigkeit privater Normgebung wirft die Frage auf, ob und inwiefern das formale Recht dazu beitragen kann, die Transparenz der emergenten Ordnungsstrukturen zu steigern und einen Rahmen bereitzustellen, in welchen die neuen Verantwortlichkeiten und Verhaltensstandards wirksam eingebunden werden können.

Einer verbreiteten Sichtweise zufolge ist es um die Chancen für eine solche Integration eher schlecht bestellt; Gesellschafts- und Staatenwelt hätten sich bereits so sehr gegeneinander verselbständigt, dass mit der Entstehung neuer Steuerungsformen im Überschneidungsfeld beider kaum zu rechnen sei. In der Politikwissenschaft ist es vor allem das sog. dualistische Modell Rosenaus (1992), das die Debatte prägt; in der heutigen Welt bestünden viele staatliche Institutionen bloß noch als leere Hülse, während es die Akteure der nicht-staatlichen Sphäre immer besser verstünden, sich staatlichem Einfluss zu entziehen. Zwar sei der transnationale Raum Entstehungsort vielfältiger Problemlösungsmechanismen unterschiedlichster Herkunft, deren Zahl in die Millionen gehe, ein brauchbares Instrumentarium für die Koordination dieser Mechanismen sei jedoch nicht in Sichtweite. Auch in den Rechtswissenschaften finden sich Tendenzen, das Verhältnis beider Sphären als eine Konstellation der Inkommensurabilität zu konzipieren; das informelle Praxisrecht der Weltgesellschaft verkörpere ein Normsystem, »dessen Rechtsquelle nicht nur unabhängig von den nationalen Rechtsordnungen ist, sondern sich gerade gegen Praktiken der Nationalstaaten richtet « (Teubner 2000, S. 250), und die Kategorien des positiven Rechts enden nach dieser Lesart genau dort, wo der Bereich der transnationalen Normstrukturen beginnt; »in sich hochrational, aber irrational in Bezug auf die ganze Gesellschaft« (a.a.O., S. 270) sei das Recht zu sehr seinem eigenen Code verhaftet, um sich den emergenten Strukturen zu öffnen.

Nun sieht es nicht so aus, als sei mit dieser Einschätzung das letzte Wort zum Verhältnis beider Sphären gesprochen. So hatte Max Weber schon zu Beginn des 20. Jahrhunderts die Prognose aufgestellt, mit dem Wachstum des Güterverkehrs komme es zu einer Zunahme informeller Normbildungs- und Selbstbindungstatbestände, und mit diesen zu zahlreichen neuen Anforderungen an die Rechtspraxis, denen diese sich nicht entziehen könne $\left(1980^{5}\right.$, S. 506). Dass die Rückbindung an geltendes Recht grundsätzlich gelingen kann, zeigen auch 
die Erfahrungen, die im innerstaatlichen Kontext während der letzten Jahrzehnte mit privaten Initiativen gemacht wurden; man denke nur an die Steuerungsinstrumente neueren $\mathrm{Zu}$ schnitts, wie sie unter den Begriffen der »regulierten Selbstregulierung « und des »reflexiven Rechts« diskutiert werden (vgl. Schmidt-Preuss 1998). Von einer bruchlosen Übertragung dieser Regelungsformen auf die transnationale Ebene kann zwar nicht die Rede sein - nicht zuletzt aufgrund des Fehlens einer »Weltregierung « oder einer sämtliche Erdteile überspannenden »Weltgerichtsbarkeit« - , übertragbar sind aber viele der Argumente zur Flexibilität und Offenheit des formalen Rechts und seiner Tendenz, die Grenzziehung zwischen rechtsrelevanten und nicht-relevanten Sachverhalten immer wieder neu zu justieren.

Realistischer als die Annahme eines Auseinanderdriftens der verschiedenen Normstrukturen ist daher die Vorstellung vielfältiger Wechselwirkungen; eine Sichtweise, die von manchen Autoren mit dem Begriff der »Interlegalität« akzentuiert wird (Günter/ Randeria 2001, S. 31) und die den Ausgangspunkt eines viel versprechenden Untersuchungsprogramms darstellt. $\mathrm{Zu}$ den Zielen dieses Ansatzes zählt die Rekonstruktion der privaten Steuerungsarrangements in ihrer Funktionsweise sowie die Erkundung, inwieweit sie Anschlüsse im positiven, staatlich gesetzten Recht auslösen; die Klärung, in welchen Rechtsgebieten solche Anschlüsse auftreten, sowie die Beantwortung der Frage, was die Entwicklungsgesetzlichkeiten sind, die das Recht an einzelnen Stellen über sich selbst hinaustreiben. Die Ausleuchtung dieses Fragehorizontes setzt eine interdisziplinäre, juristisch-soziologische Zugangsweise voraus, wobei den Methoden der rekonstruktiven Sozialforschung für die Integration beider Disziplinen eine Schlüsselstellung zukommt. Statt sich, wie in vielen bisherigen Beiträgen, mit einem eher kursorischen Überblick zu den verschiedenen selbstregulativen Elementen zu begnügen, gilt es, sich systematisch ins Innere der Steuerungsarrangements vorzuarbeiten und präzise zu ermitteln, wie die Verantwortlichkeiten zwischen den verschiedenen Akteuren und Handlungseinheiten in der Realität konkret verteilt sind.

Wo dies gelingt, ist nicht nur eine wichtige Vorarbeit für die anschließende Durchsicht des formalen Rechts auf mögliche Anschlussstellen geleistet - ein Schritt, der ebenfalls starke empirische Anteile enthält, da viele Innovationen, bevor sie die offizielle Normgebungsebene erreichen, auf der Ebene der Rechtsanwendung erprobt und vorbereitet werden -, die detaillierte Rekonstruktion der privaten Steuerungssysteme kann auch dazu beitragen, der Rechtspraxis die oft dringend benötigte empirische Expertise bereitzustellen und hierdurch die Wirklichkeitsnähe und Sachadäquanz der Rechtsentscheidungen zu steigern. Auf eine knappe Formel gebracht handelt es sich bei dem skizzierten Ansatz um eine >Rechtssoziologie des emergenten Rechts als Rechtssoziologie für das geltende staatliche Recht<, und es kann die Prognose gewagt werden, dass die Bedeutung dieser Forschungsrichtung unter den Bedingungen des heutigen, globalen Rechtspluralismus weiter zunehmen wird. Im Folgenden soll der skizzierte Ansatz auf einen konkreten Untersuchungsfall angewandt werden, nämlich die Entstehung grenzübergreifender Privatregulative zu Umweltschutz und Sicherheit in multinationalen Unternehmen, eingegrenzt auf die acht größten Konzerne der deutschen Chemiebranche. ${ }^{2}$

2) Die Stichprobe besteht aus den Unternehmen BASF, Bayer, Beiersdorf, Degussa, Henkel, Merck, Wacker sowie der deutschen Höchst-Nachfolgerin Celanese. Vergleiche mit kleineren Konzernen, Konzernen anderer Branchen und/ oder anderer Herkunftsländer wären prinzipiell wünschenswert, müssen aber auf einen späteren Zeitpunkt verschoben werden. Die Beschränkung auf einen relativ homogenen Akteurskreis entspricht dem Ziel einer möglichst realistischen und praxisnahen, an konkreten Problemen orientierten Analyse der emergenten Steuerungsarrangements. 


\section{Umweltschutz im multinationalen Unternehmen - neue Steuerungsformen als Ergebnis kollektiven Lernens}

Wurde Globalisierung lange Zeit gleichgesetzt mit einer Tendenz der Angleichung der verschiedenen Länder und Erdteile, so liegt der Schwerpunkt heute stärker auf Aspekten der Fragmentierung (statt vieler: Robertson 1998) - die aktuellen Umbrüche in der Weltgesellschaft basieren auf Prozessen der Entgrenzung, die mit der Entstehung neuer Grenzen und Bruchstellen einhergehen. Dieses spannungsreiche Nebeneinander von Homogenisierung und Fragmentierung prägt auch viele der Probleme, wie sie im Zusammenhang mit der wachsenden Bedeutung multinationaler Konzerne diskutiert werden. Zwar tragen die Aktivitäten der Global Players dazu bei, auch die weniger entwickelten Länder immer intensiver in den Weltmarkt einzubeziehen, gleichzeitig kommt aber in der Tatsache, dass fast alle dieser Unternehmen aus der OECD-Welt stammen, eine grundlegende Asymmetrie zum Ausdruck: Die transferierten Technologien werden bis auf wenige Ausnahmen in den Stammländern entwickelt, und die Fähigkeit, selbst etwas Gleichwertiges zu entwickeln, bleibt für viele Empfängerländer in unerreichbarer Ferne. Schwer zu erreichen und nur in langwierigen Nachholprozessen aufzubauen ist auch das Gefüge an Rahmenbedingungen zur institutionellen Einbindung dieser Produktionsprozesse, wie es sich in den >alten< Industrienationen parallel zum technischen Fortschritt herausgebildet hat, das in den Entwicklungsländern jedoch meist noch deutlich in den Anfängen steckt.

So weisen die Umweltrechtssysteme vieler Entwicklungs- und Schwellenländer trotz verschiedener Modernisierungserfolge und trotz der nicht zu unterschätzenden »Dynamisierung der Umweltpolitik durch Globalisierung « (Weidner/ Jänicke 2001, S. 307) zahlreiche Regelungslücken und Steuerungsdefizite auf (Desai 1998 m.w.N.). In Afrika ist die Rechtslage von der Dominanz veralteter, vorwiegend auf Verteilungs- und Rohstoffaspekte zugeschnittener Gesetze geprägt, die der Problematik industrieller Umweltbelastungen kaum Rechnung tragen. In vielen Ländern Asiens, Lateinamerikas und Osteuropas verfügt man zwar über Spezialgesetze zu einzelnen Problemfeldern wie Gewässerschutz und Luftreinhaltung, andere Gesetze, etwa zur (Sonder-)Müllentsorgung oder zum Bodenschutz, fehlen jedoch. Aber auch dort, wo sich die umweltrechtlichen Regelungen auf gesetzlicher Ebene zu einem tragfähigen Netz zusammenfügen, mangelt es häufig an dem nötigen Unterbau an normkonkretisierenden Standards (vgl. Gündling 1998). Hinzu kommen die viel beklagten Vollzugsdefizite aufgrund von Korruption und fehlender behördlicher Kapazitäten.

Einer populären Auffassung nach hängen beide Aspekte, die Internationalisierung der Produktion und die Regelungslücken in den Entwicklungs- und Schwellenländern, eng zusammen - während die Unternehmen aus Kostengründen Länder mit niedrigen Umweltstandards bevorzugen, sehen diese sich gezwungen, von einer Verschärfung ihrer Anforderungen entweder abzusehen oder diese sogar immer weiter abzusenken (vgl. Leonard 1988 m.w.N.). Nun ist zwar richtig, dass die mit den Aktivitäten multinationaler Konzerne verbundenen Schäden in der Vergangenheit ein Ausmaß angenommen haben, wie es an den Heimatstandorten kaum denkbar gewesen wäre; dies auf eine planmäßige Ausnutzung der niedrigeren Standards zurückzuführen, verfehlt jedoch in vielen Fällen die eigentliche Problematik. In vielen Branchen, insbesondere bei kapital- und wissensintensiven Investitionen, sind die Firmen in erster Linie an einer dauerhaften und skandalfreien Geschäftstätigkeit interessiert, und die Betriebe werden mit Umweltapparaturen ausgestattet, die, wenn auch nicht in jedem Punkt auf dem Stand der Technik, doch ein vertretbares Schutzniveau ermöglichen. Die Entstehungsstruktur der genannten Probleme ist weniger in einem besonders skrupellosen Verhalten der Konzernspitze zu suchen, als vielmehr in einer breiten Palette von Fehlentscheidungen und Fehlbedienungen vor Ort, die eher auf einen gewissen Kontrollverlust hindeuten. 
War man in den Stammhäusern der Konzerne lange Zeit der Meinung, für einen sicheren und umweltverträglichen Betrieb der ausländischen Produktionsstätten genüge es, diese mit der nötigen Grundausstattung und allen zugehörigen Betriebsanleitungen zu beliefern, so ist diese Vorstellung durch das gehäufte Auftreten von unerwarteten Missständen inzwischen nachdrücklich in die Krise geraten. Wichtige Impulse für die Revision des alten, dezentralen Konzernmodells gingen von der Katastrophe von Bhopal im Jahr 1984 aus; mindestens ebenso wichtig war aber das breite Spektrum von eher >kleinformatigen< Fahrlässigkeiten und Betriebsfehlern über die Jahre, durch deren gehäuftes Auftreten immer evidenter wurde, dass die Zentrale, um ein Mindestmaß an Normalität aufrechtzuerhalten, aktiv Verantwortung übernehmen und das operative Geschehen vor Ort in ein enges Netz von Kontrollen einbinden muss. Erscheinungen wie die Missachtung aller Wartungs- und Instandhaltungsaspekte oder Fehler beim Betrieb einer Kläranlage, die deren Funktionsfähigkeit beeinträchtigen oder sogar völlig zunichte machen, all dies ist ja nicht nur unter einem ethischen und ökologischen Vorzeichen problematisch, Erscheinungen wie diese sind auch geeignet, das Vertrauen in die Funktionsfähigkeit der gesamten Konzernorganisation grundlegend in Frage zu stellen.

Das Fehlen tragfähiger Regelungsmechanismen vor Ort erzeugt somit einen gewissen Problemdruck auf Seiten der Unternehmensleitung, und gleichzeitig belasten die auftretenden Missstände auch das Verhältnis zwischen den Konzernen und der Öffentlichkeit. Es entsteht eine Situation, in der Fragen der Legitimität immer deutlicher von Kriterien der bloßen Rechtskonformität abgekoppelt werden, d.h. die pure Einhaltung aller formalrechtlichen Anforderungen erweist sich für die Akzeptanzfähigkeit wirtschaftlichen Handelns zunehmend als ungenügend, vielmehr sehen sich die Konzerne mit der Erwartung konfrontiert, den Stand des rechtlich Gebotenen in vielen einzelnen Punkten eigeninitiativ zu überschreiten. ${ }^{3}$ Wie beide Faktoren - der Einfluss einer kritischen Öffentlichkeit und Lernprozesse aufgrund neuer und krisenhafter Erfahrungen - in ihrem Verhältnis zueinander zu gewichten sind, ist eine empirisch nur schwer zu beantwortende Frage; grundsätzlich gilt, dass das Selbstverständnis einer Firma nicht durch jede beliebige Kritik zu erschüttern ist, dass durch den Druck der Öffentlichkeit aber dort, wo die Missstände auch aus der Sicht des Praktikers ein beunruhigendes Ausmaß angenommen haben, ein zusätzlicher Anreiz geschaffen wird, die Anomalien im Inneren ernst $\mathrm{zu}$ nehmen und ihnen mit speziellen Organisations- und Restrukturierungsmaßnahmen entgegenzuwirken.

Im Ergebnis konstituieren die beschriebenen Steuerungs- und Legitimationsprobleme für die Verantwortlichen in den Führungsspitzen der Konzerne das, was man vielleicht als »kollektiven Erfahrungsraum « (Mannheim 1980, S. 272) bezeichnen könnte; eine Problemlage, mit der mehrere Akteure sich in einer ganz ähnlichen Weise konfrontiert sehen, und die geeignet ist, Prozesse des gegenseitigen Lernens und Steuerungsinitiativen von ganz ähnlicher Grundstruktur auf den Weg zu bringen, auch in Abwesenheit einer regulativen Zentralinstanz oberhalb der einzelnen Konzerne. Statt nur punktuell, in einzelnen, besonders fortschrittlichen Firmen aufzutreten, prägen die anvisierten Steuerungssysteme den Verhaltensstandard ganzer Praxisfelder; eine Beobachtung, die auch für die rechtliche Verarbeitung der Regulative folgenreich sein kann. Bei der empirischen Rekonstruktion, deren Ergebnisse im Folgenden vorgestellt werden sollen, wurde diesem Aspekt durch die Eingrenzung der

3) Die politische Relevanz der Problematik zeigt sich auch an der Erarbeitung verschiedener Verhaltenskodizes durch internationale Organisationen wie die UNO, die ILO und die OECD, deren Leitlinien erst kürzlich aktualisiert und in eine auch unter Umweltaspekten sehr anspruchsvolle Version gebracht wurde (vgl. Böttger 2002, S. 133 ff). Als Steuerungsinstrumente sind diese Initiativen aufgrund ihrer Unverbindlichkeit zwar eher irrelevant, sie bringen aber doch die normativen Erwartungen der internationalen Gemeinschaft an die Global Players in gültiger Weise zum Ausdruck. 
Stichprobe auf eine relativ homogene Merkmalsgruppe und durch systematische Vergleiche zwischen den einzelnen Firmen Rechnung getragen, um diejenigen Strukturen freizulegen, die den gemeinsamen Verhaltensstandard, gewissermaßen den Stand der Praxis des betreffenden Akteurskreises konstituieren.

\section{Firmenkodizes - Audits - Standards. Zur Anatomie der privaten Steuerungssys- teme}

Die Steuerungssysteme der Konzerne bestehen aus mehreren, eng zusammenhängenden Komponenten von unterschiedlicher Funktion. Gewissermaßen die äußere Schicht bilden diejenigen Elemente, die speziell für den Kontakt mit der Außenwelt geschaffen wurden, während viele andere Bestandteile, darunter die internen Ablaufstrukturen, Kontrollsysteme und operativen Standards, sich den Augen der Öffentlichkeit weitgehend entziehen und nur im Zuge einer sukzessiven Einarbeitung in die Vorgänge im Inneren der Konzerne herauspräpariert werden können. Jede der einbezogenen Firmen veröffentlicht eigene Umweltleitlinien, die einer gründlichen, qualitativ-soziologischen Auswertung unterzogen wurden, wobei neben einer Rekonstruktion der durch die Texte eröffneten Interaktions- und Sinnstruktur auch systematische inhaltsanalytische Vergleiche durchgeführt wurden. Ferner verfügt jede der Firmen über eigene Spezialorgane, meist unter der Bezeichnung »Konzernbeauftragter für Umweltschutz und Sicherheit«, die mit der Überwachung der einzelnen Konzernteile beauftragt sind und mit deren Mitarbeitern mehrere problemzentrierte Interviews durchgeführt wurden $(\mathrm{N}=14)$; auch dieses Material wurde einer rekonstruktiv-soziologischen Auswertung auf der Basis einzelner Passagen und zahlreicher Kreuzvergleiche zwischen den Interviews zugeführt. Bei genauerer Einarbeitung stößt man in jeder der Firmen auf ein enges Geflecht organisatorischer und technischer Standards, die zum Teil in konzerninternen Dokumenten und Richtlinien festgeschrieben sind, zum Teil aber ebenfalls, da der Formalisierungsprozess bislang noch deutlich in den Anfängen steckt, aus den Interviews mit den Praktikern erschlossen werden müssen. Für die Darstellung der Ergebnisse empfiehlt es sich, die Konturen der Steuerungsarrangements gleichsam im Durchgang von >außen< nach >innen< nachzuzeichnen, beginnend mit den außengerichteten Firmendokumenten.

\subsection{Unternehmensleitlinien}

Für die Interpretation der Umwelt- und Sicherheitsleitlinien ist wichtig, dass es sich hierbei, auch wenn dies nicht immer explizit hervorgehoben wird, um Selbstverpflichtungen handelt. Die Texte tragen Überschriften wie »Grundsätze für Umweltschutz und Sicherheit«, »Weltweite Standards« oder auch »Umweltleitlinien - unser >Grundgesetz««; eine Analogie, mit der die Verfasser die Rechtsähnlichkeit der Dokumente in einer fast provokativ anmutenden Weise selbst unterstreichen. Der Umfang beträgt in der Regel 1-3 Textseiten; dem eigentlichen Leitlinienteil vorgeschaltet ist meist eine kurze Vorbemerkung, in der die Bestimmungen in einen breiteren gesellschaftlichen Kontext gestellt werden, und in der Zusammenhänge zwischen Umweltschutz und Unternehmenserfolg hervorgehoben werden. Auch der eigentliche Leitlinienteil ist meist sehr knapp formuliert; die Bestimmungen beziehen sich neben Aspekten der Gefahrenabwehr auf Fragen der Vorsorge und Emissionsreduzierung; häufig enthalten sie die Maxime der kontinuierlichen Unterweisung und Schulung aller Mitarbeiter; in einigen Exemplaren wird auf die Einrichtung konzernübergreifender Managementsysteme hingewiesen, und in vielen Fällen wird neben der Pflicht zur umweltverträglichen Gestaltung aller Abläufe im Inneren auch eine Pflicht zur Überwachung der Geschäftspartner statuiert.

Die Firmenkodizes sind ein fast paradigmatisches Beispiel dafür, wie es privaten Akteuren gelingt, aus eigener Kraft Normstrukturen von erheblicher Bindungswirkung zu etablieren. 
Indem die Konzerne sich mit eigenen Verhaltenskodizes an die Öffentlichkeit wenden, nehmen sie eine verbindliche Abgrenzung gegenüber den Versäumnissen der Vergangenheit vor; die Texte erheben den Anspruch auf Glaubwürdigkeit, und sie tun dies (in einer nur scheinbar paradoxen Bewegung), indem die Integrität und Glaubwürdigkeit der Konzerne öffentlich mit der Hypothek der Befolgung der statuierten Pflichten belastet wird. Wie bei jedem Sprechakt vom Typus einer Eigenobligation werden durch die Leitlinien normative Fakten geschaffen (vgl. Austin 2002; Rolf 1997); Abweichungen können später nicht mehr als einfache Kurskorrektur hingestellt werden, vielmehr erlangen sie die Qualität einer Verletzung berechtigter normativer Erwartungen. Drei Aspekte sind es, die für eine genauere Erörterung der Textstruktur von Bedeutung sind; erstens die Weise, in der die Verfasser sich zur Sphäre des geltenden, staatlichen Rechts ins Verhältnis setzen; zweitens die Frage nach der Sachhaltigkeit der Bestimmungen und ihrer Anwendbarkeit auf konkrete Fälle, sowie drittens die Verteilung von Eingriffs- und Weisungsbefugnissen im Verhältnis zwischen Mutter- und Tochtergesellschaft.

Hinsichtlich der Bezüge zum geltenden Recht ist festzuhalten, dass bei der normativen Verankerung der Selbstverpflichtungen offenbar nicht auf vorhandene juristische Formate rekurriert wird (etwa: Vertrag; Satzung), ihre Geltungsgrundlagen sind durchaus >autonom‘; eine Form der Herstellung von Ordnung, die als wichtiges und Sozialität stiftendes Potential in den allgemeinen Strukturen der Lebenswelt verankert ist. Indem die Akteure Selbstbindungen eingehen, werden sie »von potentiellen Subjekten zu manifesten« (Oevermann 1983, S. 237); das zukünftige Verhalten wird für andere antizipierbar und kann von diesen, auf der Basis eines gewissen Vertrauensvorschusses, zur Grundlage des eigenen Handelns gemacht werden. Mit einer Aushöhlung staatlicher Entscheidungsbefugnisse ist dies nicht gleichzusetzen - sinnlogisch können die Leitlinien sich nur auf einen Bereich oberhalb des rechtlich Gebotenen beziehen, was durch Formulierungen wie »als notwendig Erkanntes nehmen wir auch ohne gesetzliche Verpflichtungen oder behördliche Auflagen in Angriff « auch adäquat auf den Begriff gebracht wird. Käme in den Texten die Absicht zum Ausdruck, neben verschiedenen Mindestanforderungen und Optimierungsprinzipien auch die Obergrenze festzulegen, ab welcher weitere Umweltmaßnahmen nicht mehr als erforderlich anzusehen sind, so wären sie als Mittel der Vertrauenswerbung gescheitert; dem äußeren Anschein nach ein Versuch, sich um eine neue und tragfähige Positionierung im öffentlichen Raum zu bemühen, hätte man es in Wirklichkeit mit einer Usurpation gesetzgeberischer Kompetenzen, gewissermaßen mit einer Anmaßung fremder Souveränität zu tun. ${ }^{4}$

Die Werbung um öffentliches Vertrauen setzt ferner voraus, dem Empfänger einen ungefähren Eindruck zu vermitteln, worauf er sein Vertrauen überhaupt gründen soll, d.h. die Leitlinien müssen, trotz ihres programmatischen Charakters, konkrete Informationen über die anstehenden Probleme und die entsprechenden Lösungen enthalten. Wie von Kritikern häufig moniert wurde, rangieren die Firmenkodizes meist auf einem sehr hohen Abstraktionsniveau (Vorwurf der »Öko-Rhetorik«; vgl. Fuchs 1997); sobald man die Texte freilich etwas gründlicher interpretiert und sie mit möglichen Anwendungsfällen konfrontiert, wird schnell deutlich, dass ihr Aussagegehalt präzise genug ist, um zumindest einzelne, stark schädigende Praktiken eindeutig in den Bereich des Unzulässigen zu verweisen (wobei me-

4) Dass auch der umgekehrte Fall, die explizite Zusicherung der Beachtung des geltenden Rechts, letztlich zu einer paradoxen Sinnstruktur führt, sei hier nur der Vollständigkeit halber angefügt (detailliert Herberg 2001); die Paradoxie besteht darin, dass hierbei etwas, das die Konzerne ohnehin tun müssen, nämlich die rechtlichen Anforderungen zu erfüllen, als spezielle Sonderleistung dargestellt wird. Legalitätsgelöbnisse dieser Art treten in einzelnen Leitlinienexemplaren tatsächlich auf - ein Beleg für die Schwierigkeiten, unter heutigen Bedingungen zu einem ausgewogenen Verhältnis zwischen den verschiedenen Steuerungsbeiträgen staatlicher und privater Provenienz zu gelangen. 
thodisch streng darauf zu achten ist, nur diejenigen Bedeutungen herauszufiltern, die tatsächlich als zwingende Implikation in den Texten enthalten sind).

So kann die Aussage »Wir erzeugen Produkte, die sicher herzustellen, sicher zu verwenden und sicher zu entsorgen sind « im Rahmen einer Selbstverpflichtung nichts anderes bedeuten als >alle unsere Produkte sind sicher herzustellen, sicher zu verwenden und sicher zu entsorgen $<$ (die Version > wir erzeugen unter anderem solche Produkte < wäre sinnlogisch undenkbar, da sie den Leser mit der Zumutung konfrontieren würde, etwas als spezielle Sonderleistung zu werten, das ohnehin erfolgt). Ein Verstoß läge etwa dann vor, wenn elementare Schutzvorkehrungen im Umgang mit bestimmten Substanzen, die allgemein als umweltoder gesundheitsgefährdend bekannt sind, in einzelnen Produktionsstätten des Konzerns fehlen. Zu den Implikationen der Textstelle gehört auch, konzernintern über effektive Formen des Informationsmanagements zu verfügen, um neue Erfahrungen mit dem Schädigungspotential bestimmter Substanzen, die an einem der Standorte zu Lernprozessen führen, zügig an alle anderen Konzernteile zu übermitteln, um auch dort die nötigen Gegenmaßnahmen zu treffen.

Was den dritten Punkt betrifft, die Frage nach der Steuerungsfähigkeit der Konzerne im Inneren, so steht bei Versprechen, die Individuen einander im Alltag geben, deren Selbststeuerungs- und Zurechnungsfähigkeit in der Regel nicht in Frage, bei den öffentlichen Selbstverpflichtungen korporativer und über mehrere Erdteile verstreuter Akteure hingegen sehr wohl. Es ist eine wichtige sinnlogische Implikation der Leitlinien, dass die Unternehmensleitung als Urheber der Texte in der Lage sein muss, Verantwortung für die Einlösung der Zusagen zu übernehmen und Einfluss auf die Vorgänge in den verschiedenen Konzernteilen auszuüben; eine Bedingung, die angesichts der in der Chemiebranche üblichen 100\%igen und mehrheitlichen Beteiligungs- und Eigentumsverhältnisse auch keineswegs abwegig erscheint. Um die Wirkung einer glaubwürdigen Selbstverpflichtung zu erzielen, dürfen die Texte keinerlei Anhaltspunkte dafür enthalten, dass ihre Einhaltung ins freie Ermessen der Mitarbeiter gestellt sei; träfe dies zu, so könnten die Leitlinien allenfalls als internes Rundschreiben, nicht aber als Mittel der Vertrauenswerbung im Außenverhältnis Verwendung finden. Dies muss keineswegs zu einer Bündelung sämtlicher Entscheidungen am Stammsitz des Unternehmens führen, es muss aber gewährleistet sein, dass gravierende Missstände, sollten sie in einer der Tochtergesellschaften auftreten, mit einer gewissen Wahrscheinlichkeit entdeckt und zum Anlass geeigneter Gegenmaßnahmen werden.

\subsection{Auditierung}

Auch bei der Analyse der internen Kontrollsysteme empfiehlt es sich, zunächst das Verhältnis zum geltenden Recht zu klären, sodann Überlegungen zur Sachhaltigkeit und Prüftiefe der Kontrollen anzustellen und schließlich das Verhältnis zwischen Mutter- und Tochtergesellschaft nachzuzeichnen, wie es sich in den Aussagen der Interviewpartner dokumentiert. Zum Tätigkeitsprofil der Umweltabteilungen auf Konzernleitungsebene ist vorauszuschicken, dass diese schon in der Phase der Neuerrichtung einer Produktionsstätte auf die Ausstattung mit allen nötigen technischen Vorkehrungen hinwirken, was institutionell in einer gewissen Veto-Macht gegenüber den einzelnen Geschäfts- und Länderbereichen verankert ist. Zum Aufgabenspektrum zählt ferner die Anleitung der Auslandsbetriebe bei allen auftretenden Problemen, in besonders gravierenden Fällen auch in Form einer Beratung vor Ort, die von den Betrieben jederzeit angefordert werden kann - »wenn irgendwo ein Betrieb kommt und sagt, Leute, ich fahre nicht mehr weiter, ich will sofort eine Bestandsaufnahme«, so die Aussage in einem der Interviews, »dann sind wir quasi über Nacht da (Interv. 13.12.2000). Bewegt sich dies noch weitgehend im Rahmen des alten, eher dezentralen Konzernmodells (die Zentrale als Technik- und Wissensgeber), so kommt die entscheidende Ak- 
zentverschiebung in dem folgenden Aufgabenschwerpunkt zum Ausdruck: In einem regelmäßigen, etwa 2-3 jährigen Turnus wird an sämtlichen Standorten eine gründliche Durchsicht aller umweltrelevanten Apparaturen und Abläufe durchgeführt, deren Ergebnisse in einem detaillierten Bericht festgehalten und mit speziellen Sanierungsforderungen verknüpft werden.

Was den Bezug zum geltenden Recht betrifft, so ist das Gesellschafts- und Konzernhaftungsrecht der verschiedenen Länder der Welt weit davon entfernt, Steuerungsaktivitäten wie diese verbindlich vorzuschreiben. Bei den Tochtergesellschaften handelt es sich um juristisch selbständige Einheiten, für deren Versäumnisse die Muttergesellschaft höchstens dann einstehen muss, wenn die dortige Geschäftsführung aufgrund einer übermäBigen Einschränkung ihrer Entscheidungsautonomie zu einer selbständigen Erfüllung ihrer Aufgaben nicht mehr im Stande war. Gesetzesentwürfe wie der US-amerikanische Foreign Environmental Practices Act, der sämtliche amerikanische Unternehmen bei allen Auslandsaktivitäten bestimmten Mindeststandards unterwerfen sollte (vgl. Neff 1990), haben sich rechtspolitisch als Eintagsfliege erwiesen - wohl nicht zuletzt aufgrund der Unmöglichkeit, für den nötigen Unterbau an administrativen, grenzübergreifenden Implementationssystemen zu sorgen. Wenn die Konzerne nun ihrerseits darangehen, entsprechende Kontrollstrukturen zu etablieren, dann übernehmen sie die Rolle eines globalen Exekutivorgans, was teilweise auch mit quasi-polizeilichen und quasi-kriminalistischen Elementen verbunden sein kann; dies jedenfalls dann, wenn auch solche Missstände in den Blick gelangen, die von Ausführenden nicht oder nur widerwillig zugegeben werden, und wenn den Kontrolleuren effektive Eingriffsmöglichkeiten bereit stehen.

Tatsächlich enthält das Material zahlreiche Hinweise, dass es während der Vor-OrtKontrollen (»Audits«) grundsätzlich gelingen kann, auch versteckten Problemen auf den Grund zu gehen. Die Konzernbeauftragten agieren als »Spezialisten für die Entdeckung des schwer Entdeckbaren« (Oevermann u.a. 1985, S. 135) mit der Aufgabe, den trügerischen Schein von Anlagen, die nach außen einen noch so gepflegten Eindruck machen mögen, zu durchbrechen. Die Auditoren stützen sich hierbei auf eigene technische Sachkenntnisse - die meisten unter ihnen haben selbst langjährige Erfahrungen als Betriebsleiter hinter sich - sowie auf eine Art $\gg$ Fehlerkunde<, ein Wissen um besonders häufig auftretende Versäumnisse und Betriebsfehler. Ausgangspunkt der Audits ist meist eine Durchsicht der innerbetrieblichen Handbücher, Ablaufprozeduren, Wartungspläne etc., gründliche Nachforschungen in Form von Betriebsbegehungen werden hierdurch jedoch nicht überflüssig - vielmehr scheint man bei den Inspektionen gerade denjenigen Aspekten besonders gründlich nachzugehen, die im Rahmen der Dokumentenanalyse zunächst unproblematisch erscheinen. ${ }^{5}$ Eine lückenlose Defizitanalyse ist mit diesem Instrument zwar nicht möglich, durch eine geschickte

5) Die Überfokussierung auf formelle Aspekte, wie die Auditoren sie bei manchen Fachkollegen beobachten (vor allem: Kollegen aus der Zertifizierungsbranche), markiert gewissermaßen den »negativen Gegenhorizont « (Bohnsack 1993, S. 41) der eigenen Tätigkeit. Interviewer: »Aber die Frage ist doch, was kann man mit bloßem Auge erkennen? Im Grunde ist man doch angewiesen auf den Gesamteindruck, den das Ganze vermittelt.«-Befragter 1: »Das ist das, was mein Kollege zu Anfang gesagt hat. Dadurch, dass wir hier eine Mischung sind aus operativ und strategisch Tätigen, und zwar jeder für sich, und nicht diese Trennung haben, >da ist jetzt eine Audit-Abteilung, die macht irgendwas Theoretisches<, ist das einfach ein Erfahrungswert. « (...) - Befragter 2: Und das ist unser Konzept, dass wir uns quer mit Informationen versorgen. Dass wir uns nicht spezialisieren auf einen Bereich und dann die Bodenhaftung - das ist meine ganz große Angst, dass wir die Bodenhaftung verlieren, sondern, dass wir schon immer versuchen zu sehen, wie läuft's denn wirklich hier vor Ort« (Interv. 15. 1. 2001). Der Gefahr einer Verselbständigung der formellen Aspekte ist, wie hier von den Auditoren sehr nachdrücklich zum Ausdruck gebracht wird, kontinuierlich entgegenzusteuern; gelänge dies nicht, müsste die eigene Praxis letztlich als gescheitert betrachtet werden. 
Auswahl der Beobachtungsgegenstände scheint es aber zu gelingen, auch und gerade solche Auffälligkeiten zu identifizieren, die Symptome für Dysfunktionen weiterreichenden Charakters darstellen.

Kommt bereits in der Befugnis der Konzernbeauftragten, einzelne Problemaspekte verbindlich als Verstöße gegen die geltenden Normen und Regeln zu definieren, ein gewisses hierarchisches Moment zum Ausdruck, so wird dies durch die bestehenden Eingriffsund Weisungsbefugnisse noch zusätzlich untermauert. Dass diese autoritativen Elemente bei der öffentlichen Außendarstellung der Unternehmen nur sehr dezent zur Sprache kommen, kann verschiedene Gründe haben, von der Angst vor neuen juristischen Risiken bis hin zu dem Bemühen, sich nach außen einen möglichst konfliktfreien Anstrich zu geben. In der Praxis spielen die Durchgriffsrechte der Auditoren jedoch eine wichtige Rolle, und in besonders gravierenden Fällen reichen sie bis zur Verhängung von Stilllegungsbefugnissen mit sofortiger Wirkung: »Wenn wir wo hinkommen, wo Gefahr im Verzug ist, sagen wir, bitte stoppt das sofort«, eine Aussage, die sich in ähnlicher Form auch in sämtlichen anderen Interviews wieder findet (wobei das Wörtchen »bitte« an dieser Stelle eine gewisse Rollendistanz zum Ausdruck bringt, die wiederum dem Spannungsverhältnis zwischen der Autonomie der Konzernteile und dem Steuerungsanspruch der Zentrale geschuldet ist).

Es ist kein Widerspruch zu dem Gesagten, dass die Auditoren sich über weite Strecken um einen möglichst kooperativen und konfliktfreien Vollzugsstil bemühen (»Wir wollen von der Sinnhaftigkeit überzeugen, dann läuft das auch von allein«, Interv. 15.1.2001). Häufig sind die Kritikpunkte und Anweisungen der Auditoren auch von einer Plausibilität, die den Verantwortlichen vor Ort unmittelbar einleuchtet - nicht zuletzt deshalb, weil viele Missstände weniger auf ein übertriebenes Interesse an Kostenersparnis, als vielmehr auf Erscheinungen des Organisationsversagens und auf Kommunikationsprobleme beim Technologietransfer hindeuten (etwa im Falle von Schutzvorkehrungen, die aufgrund einer falschen Montage den Schaden vergrößern, statt ihn zu verringern). Hinzugefügt sei noch, dass die Weisungsbefugnis nicht nur eine allgemeine Ermächtigung darstellt, die von den Implementeuren flexibel ausgefüllt werden kann, sondern auch mit der Verpflichtung verknüpft ist, bei Defiziten oberhalb einer gewissen Erheblichkeitsschwelle in aller gebotenen Konsequenz für Abhilfe zu sorgen und in Fällen besonderer Renitenz die Konzernspitze zu alarmieren.

Das Einflechten der verschiedenen Umwelt- und Sicherheitsbelange in das Funktionsgefüge der Konzerne geht, so das Fazit, mit einer grundlegenden Transformation der Konzernstruktur einher; statt ausschließlich mit strategischen, auf die allgemeine Marschroute bezogenen Aspekten befasst zu sein, ist die Muttergesellschaft in hohem Maße in die operativen Vorgänge vor Ort involviert. Die Verantwortungsverteilung zwischen Mutter- und Tochtergesellschaft, wie sie sich aus dem empirischen Material rekonstruieren lässt, weist der einzelnen Gesellschaft eine neue Stellung zu, wie man sie als Außenstehender und auf der Basis der Literatur zur dezentralen Konzernorganisation, zum »heterarchischen «, »polyzentrischen « oder »postmodernen « Konzern (Chandler 1966; Bartlett/ Goshal 1999; Teubner 1991) nicht unbedingt erwartet hätte - die Tochtergesellschaften sind zwar nach wie vor selbständig, aber in einem deutlich relativierten Sinn, nämlich im Sinne einer Selbständigkeit unter Eingriffsvorbehalt.

\subsection{Interne Standards}

Gibt die empirische Analyse der internen Auditierung Aufschluss darüber, wie die Implementation vonstatten geht, so steht eine genauere Untersuchung dessen, was implementiert wird, welche Kriterien hierbei zugrunde gelegt werden, noch aus. In jeder der Firmen findet sich für jeden der Problembereiche Anlagensicherheit, Arbeitsschutz, Luftreinhaltung, Abwasserreinigung und Abfallbehandlung ein engmaschiges Geflecht von Normen und Regeln mit der Funktion, für kontrollierbare Abläufe zu sorgen und die Abwälzung negativer 
Effekte auf die Allgemeinheit wirksam zu begrenzen. Für das Projekt einer Soziologie des selbst geschaffenen Rechts der Wirtschaftswelt ist die Rekonstruktion dieser Standards von besonderer Bedeutung, geben sie doch Aufschluss darüber, welches Niveau an Risikovorsorge von den Global Players nach heutigem Stand der Praxis konkret erwartet werden kann. Auch hier liegt der Fokus auf denjenigen Sorgfaltskriterien, die sich im Firmenvergleich durch einen hohen Grad an Gleichförmigkeit auszeichnen, sei es aufgrund der Ähnlichkeit der auftretenden Probleme, der kulturellen Verankerung der Unternehmen in demselben Herkunftsland oder aufgrund des horizontalen Erfahrungsaustauschs zwischen den Firmen. Der Anspruch auf eine erschöpfende Bestandsaufnahme kann hierbei selbstverständlich nicht erhoben werden, durch eine geeignete Interviewführung kann aber dafür gesorgt werden, vor allem diejenigen Verhaltensstandards in den Blick zu fassen, die auf typische und regelmäßig auftretende Missstände referieren und insofern in der Praxis eine zentrale Rolle spielen.

Grundsätzlich gilt, dass die internen Normen und Regeln sich teilweise eng an die Bestimmungen des deutschen Umwelt- und Technikrechts anlehnen, dass die Konzerne von einer detailgetreuen Übertragung deutscher Standards jedoch weit entfernt sind. Bei dem Bemühen, an sämtlichen Standorten ein einheitliches Schutzniveau oberhalb der teilweise sehr lückenhaften Anforderungen der Tätigkeitsstaaten zu etablieren, kommt dem Recht des Herkunftslandes eine wichtige Bedeutung als Quelle von Anregungen zu; Grundlage der Norm-Rezeption ist aber stets die eigenen Risikobewertung: »Wir versuchen, das, was wir gut finden am deutschen Umweltrecht, so zu neutralisieren, dass wir es übertragen können ins Ausland. Wir werden aber nicht sagen, du musst Paragraph 20 machen, bloß weil er in Deutschland da ist « (Interv. 13.12.2000). Abweichungen vom Anforderungsniveau des Herkunftslandes kommen einmal dadurch zustande, dass manche der rechtlichen Vorgaben schon für deutsche Verhältnisse als übertrieben angesehen werden (ein häufig erwähntes Beispiel sind die hohen Anforderungen an die Denitrifikation des Abwassers). Hinzu kommt, dass die Zustände in der einheimischen Industrie vieler Tätigkeitsländer den Einsatz bestimmter, besonders aufwendiger Technologien als Luxus erscheinen lassen, den man sich angesichts des hohen Wettbewerbsdrucks nicht ohne weiteres leisten kann.

Die geltenden Regeln betreffen einmal die sachtechnische Ausstattung der Standorte. Soweit es sich um die Neuerrichtung eines Werks handelt, werden die wichtigsten Festlegungen in den Stammwerken getroffen; die Veränderung, Erweiterung und Sanierung bestehender Anlagen erfolgt hingegen unter der Regie der einzelnen Auslandsgesellschaften, was sich als Quelle zahlreicher Defizite erweisen kann. Dies betrifft nicht nur Fälle, in denen notwendige Maßnahmen aus Kostengründen verzögert werden, zu den auftretenden Missständen zählt auch das Versäumnis von Instandhaltungsarbeiten, die mit wenig Aufwand und geringen Kosten $\mathrm{zu}$ bewerkstelligen wären, oder das Auftreten von in-sich-paradoxen Konstruktionen; Konstruktionen, bei denen der ursprüngliche Zweck einer Apparatur aufgrund von Montagefehlern wieder zunichte gemacht wird. Die folgenden Regeln sind eine analytische Abstraktion aus den Angaben und Belegerzählungen der Interviewpartner; sie sind in sämtlichen untersuchten Konzernen in Geltung:

- Im Umgang mit Technik ist stets der Verschleißfaktor zu berücksichtigen. Mängel in der Abdichtung der Klärbecken, der Behälter und Leitungen sind zügig zu sanieren.

- Hierbei ist auch dem Stand der Technik Rechnung zu tragen: »Früher hat man die Klärbecken gekachelt, das ist heute nicht mehr Stand der Technik« (Interv. 7.1.2002).

- Unterirdische Tanks mit bodengefährdenden Inhaltsstoffen müssen zweiwandig konstruiert und mit Leckagemeldern ausgestattet sein.

- Bei der Lagerung von Chemikalien ist darauf zu achten, zwischen bestimmten Fraktionen (etwa: Stoffe, die heftig aufeinander reagieren) bauliche Sicherheitsabstände einzuhalten. 
Tatsächlich reichen die Steuerungsarrangements bis weit auf die 'Mikroebene' des operativen Anlagenbetriebs hinunter, was angesichts des hohen Schädigungspotentials scheinbar geringfügiger Abweichungen auch kaum anders denkbar wäre. Eine Interpretation in Kategorien von »Unternehmensethik « oder »Unternehmensphilosophie«, wie in verschiedenen Beiträgen aus dem Umkreis der Betriebswirtschaftslehre (etwa: Scherer 2003; Stöckl 1996), erweist sich hier als unzulänglich, da viele der Regelungen auf tiefer liegende, in den grundlegenden Schichten technischer Vernunft verankerte Notwendigkeiten und »Basisregeln « (Cicourel 1973) verweisen, wie auch von den Interviewpartner selbst hervorgehoben wird (etwa: »Diese ganze Geschichte hat naturwissenschaftliche Hintergründe«; Interview 15.4.2002). Als schriftlich fixierte Verhaltensrichtlinien liegen die genannten Regeln nicht vor, ${ }^{6}$ was ihre Verbindlichkeit jedoch nicht schmälert, auch wenn die Implementation durch eine stärkere Formalisierung und Systematisierung der Regeln in mancher Hinsicht optimiert werden könnte (tatsächlich lassen sich in allen untersuchten Unternehmen Anstrengungen in diese Richtung beobachten). Soweit einige Kriterien zur Beschaffenheit der technischen Anlagen; der operative Kern der Regulative besteht darüber hinaus aus Standards zur Gestaltung der Betriebsorganisation, die sich ebenfalls durch einen hohen Grad an Problem- und Praxisnähe auszeichnen:

- Für einzelne Problemfelder wie Abwasser oder Abfall sind, analog zur Funktion des Betriebsbeauftragten im deutschen Umweltrecht, spezialisierte Zuständigkeiten einzurichten.

- Diese Einheiten sind mit ausreichenden Weisungsbefugnissen auszustatten: »Sie müssen auch, weil eine Kläranlage nicht Mädchen für alles sein kann, rückwärtig den Betrieben sagen können, ich nehme nicht mehr als diese Spannweite an« (Interv. 5. 12. 2001).

- Für bestimmte Tätigkeiten (z.B. Arbeiten in den Behältern) ist ein sog. Erlaubnisscheinverfahren einzuführen, um alle Gefahrenquellen schon im Vorfeld auszuschalten.

- Die Abnehmer von Sondermüll sind regelmäßig auf ihre Zuverlässigkeit zu durchleuchten: »Das mussten wir auch erst lernen, dass es wichtig ist, die End-Dienstleister zu überprüfen. Und ich muss sagen, dieses Wissen ist noch nicht überall vorhanden« (Interv. 15. 4. 2002).

So wie es soziologisch sinnvoll ist, bestimmte Regeln als grundlegende Kriterien technischer Vernunft einzustufen (etwa das Erfordernis, im Umgang mit Technik stets den Verschleißfaktor zu berücksichtigen), so stößt man bei der empirischen Rekonstruktion auch auf elementare Kriterien organisationaler Rationalität, etwa die Regel, den Grad an Fahrlässigkeiten durch geeignete Ablaufstrukturen auf ein Minimum zu reduzieren, oder das Prinzip, riskanten Alleingängen Einzelner wirksam entgegenzusteuern. Die Bestimmungen schmiegen sich eng den Problemen auf operativer Ebene an, was es nahe legt, die emergenten Ordnungsstrukturen insgesamt als pragmatische Arrangements zu charakterisieren. Anders als vor einigen Jahrzehnten, als eine Ausstattung der Auslandsbetriebe mit den nötigen Apparaturen und Bedienungsanleitungen noch als ausreichend galt, ist man dazu übergegangen, auch den gesamten Hintergrund an technischen und organisatorischen Rationalitätskriterien immer stärker in den Fokus der Unternehmenskommunikation zu rücken. Auch dort, wo die Produktionsprozesse sich aus der Sicht des Außenstehenden nicht verändern, ist hierdurch doch eine andere Konstellation entstanden als auf der Stufe der alten, fast ethnozentrisch anmutenden Vorstellung, die Funktionstüchtigkeit einer Technologie bliebe in jedem Land und an jedem Ort der Welt dieselbe.

6) Eine Ausnahme sind die Normen zur sicheren Lagerung von Chemikalien; hier greift man auf eine Empfehlung des Chemieverbandes VCI zurück - mit zahlreichen Klassifikationskriterien und quantitativen Angaben zu den nötigen Sicherheitsabständen -, die intern in den Rang einer konzernweit gültigen Vorschrift gehoben wird. 


\section{Entkoppeltes Recht? Zum Einbau der Regulative in die institutionelle Ordnung}

Die empirische Rekonstruktion erbrachte zahlreiche Einblicke in die Entstehung eines emergenten transnationalen Rechts, wie es in ähnlicher Ausprägung auch in vielen anderen Problemfeldern der globalen Wirtschaftswelt auftritt. Die Bestimmungen sind ausreichend konkret, um klare Grenzlinien zwischen zulässig und unzulässig zu etablieren, und gleichzeitig sind sie von einer Allgemeinheit, die sie als einzelfallübergreifende und für den gesamten Akteurskreis geltende Ordnung qualifiziert. Die Interviews mit den konzerninternen Kontrolleuren enthalten eine Reihe von Fällen, in denen es gelungen zu sein scheint, einzelne Schwachstellen an den Auslandsstandorten dingfest $\mathrm{zu}$ machen und innerhalb kürzester Zeit die nötigen Gegenmaßnahmen auf den Weg zu bringen. Die Stabilisierung der Normgeltung durch spezialisierte Durchsetzungs- und Erzwingungsorgane kennzeichnet die Regulative als rechtsähnliche Gebilde und para-legale Systeme, und selbst die Tatsche, dass eine endgültige und lückenlose Beseitigung aller Verstöße sich dem Bereich des Machbaren entzieht, prägt sowohl die Sphäre des >lebenden< Rechts der Wirtschaftswelt, als auch die des staatlichen Rechts. Tragen die beschriebenen Normstrukturen dazu bei, in den Lücken der bestehenden Steuerungsarchitektur verbindliche Verhaltensstandards und neue Formen der Erwartungssicherheit zu etablieren, so erzeugen sie in mancher Hinsicht doch neue Formen der Ungewissheit, vor allem mit Blick auf die Transparenz der geltenden Standards und ihre Einklagbarkeit oder Erzwingbarkeit durch Außenstehende - Probleme, die durch eine stärkere Öffnung des staatlichen Rechts für die Normentstehungsprozesse der transnationalen Sphäre zum Teil abgefedert werden können.

Tatsächlich sind, trotz des informellen Charakters der konzerneigenen Regulative, zahlreiche Fallkonstellationen identifizierbar, in denen es zu Wechselwirkungen mit dem formalen, staatlich gesetzten Recht kommt, wobei vor allem die konkreten Vorgänge auf Rechtsanwendungsebene eine zentrale Rolle spielen. Teilweise ist zu beobachten, dass die Genehmigungsinstanzen vor Ort sich selbständig über die geltenden Standards informieren und diese in ihre Behördenentscheidung inkorporieren. Eine weitere Querbeziehung liegt dort vor, wo die in der Unternehmenspraxis entstandenen Aufsichts- und Eingriffsbefugnisse neue Formen der Konzernhaftung anstoßen, bei denen weniger der übermäßige Eingriff in die Angelegenheiten der Tochtergesellschaft, als vielmehr das Fehlen geeigneter Steuerungsaktivitäten haftungsauslösend wirkt. In einzelnen Fällen können auch die außengerichteten Selbstverpflichtungen zur Grundlage von Schadensersatzforderungen werden, nämlich dann, wenn es den Anspruchsinhabern gelingt, ihr Vertrauen in die Zusagen als berechtigte und rechtlich schützenswerte Erwartungen auszuweisen.

Dass die heutige Konstellation eines globalen Rechtspluralismus für die Rechtspraxis mit zahlreichen Irritationen verbunden ist, wird von manchen Autoren prägnant mit dem Begriff der wachsenden »rechtlichen Porösität« hervorgehoben (Santos 1995, S. 473); sowohl auf der Ebene der Sachverhaltsermittlung, als auch auf der Stufe der Rechtsauslegung häufen sich die Schwierigkeiten, präzise zwischen Recht und Nicht-Recht zu unterscheiden. Unter der Regie grundlegender juristischer Kategorien wie der Figur der Verkehrsüblichkeit oder des Prinzips des Vertrauensschutzes wird der Rechtsanwender sich häufig zu einer genaueren Durchleuchtung der informellen Strukturen genötigt sehen; die Schwierigkeiten beginnen aber bereits bei der Ermittlung der geltenden Normen und ihrer inhaltlichen Reichweite. In vielen Fällen wird das Vorliegen identischer Regelungen bei einer größeren Anzahl vergleichbarer anderer Unternehmen das entscheidende Argument sein, jene auch in juristischer Hinsicht für verbindlich zu erachten, aber wie soll der Jurist an die nötigen Milieu- und Insiderkenntnisse gelangen? Wo es an verwertbarer empirischer Expertise mangelt, sieht sich die Rechtspraxis vor erhebliche kognitive Probleme gestellt, bis hin zu der Schwierigkeit, die nötige Rekonstruktionsarbeit im Alleingang leisten zu müssen. 
Weberianisch gesprochen besteht die Problematik vor allem in der Gefahr eines Auseinandertretens der formal-rationalen und der materialen Komponenten des geltenden Rechts (vgl. 19805 , S. 397). Der gesetzespositivistische Satz, was nicht explizit verboten sei, sei folglich erlaubt, gelangt in der heutigen Konstellation immer weiter an seine Grenzen; gleichzeitig käme es aber einer Preisgabe der Berechenbarkeit, Präzision und Systematik des Rechts gleich, jeden Standard, der in praktischer oder politischer Hinsicht wünschenswert erscheint, ohne alle Umschweife in den rechtlichen Diskurs zu importieren. Bewegten sich die bisherigen Überlegungen auf der Stufe einer Rekonstruktion des emergenten Rechts als Rechtssoziologie für das geltende staatliche Recht, so ist nun zu fragen, welche Formen der rechtlichen Rückbettung sich beobachten lassen; auch dies ein wichtiges Untersuchungsfeld rechtssoziologischer Forschung, das im Folgenden allerdings nur in groben Strichen umrissen werden kann.

Vieles weist darauf hin, dass auch bei den staatlichen Akteuren in Entwicklungs- und Schwellenländern ein Bewusstsein dafür entstanden ist, dass die Motivation der Konzerne zur Gründung von Auslandsstandorten und ihre Bereitschaft $\mathrm{zu}$ einem angemessenen Umweltschutzniveau einander keineswegs ausschließen - wobei behördlicherseits teilweise auch eine besondere Strenge zu beobachten ist, die mancherorts die Qualität einer Ungleichbehandlung der westlichen Firmen annimmt: »Von ausländischen Firmen verlangt das USB (chinesisches Umweltbüro) oft, die neueste Umweltschutztechnologie zu installieren, selbst wenn solche Maßnahmen die chinesischen Standards übertreffen« (Böhm 2000, S. 184). Unter material-rationalen Aspekten erscheint eine strengere Behandlung der Konzerne dadurch begründet, dass diese tatsächlich, aufgrund ihrer Finanzkraft und ihres privilegierten $\mathrm{Zu}$ gangs zur modernen Technikwelt, über sehr viel größere Problemlösungskapazitäten verfügen als die Mehrzahl einheimischer Betriebe; rein rechtlich steht dies freilich in einem gewissen Spannungsverhältnis zum Prinzip der Nicht-Diskriminierung im geltenden Wirtschaftsvölkerrecht.

Der Frage, inwieweit sich die Behörden bei ihren Entscheidungen die selbstgesetzten Standards der Corporate World zunutze machen, kommt hier besondere Bedeutung zu, da sich mit einem solchen, >reflexiven< Steuerungsmodus die Probleme eines rein mechanischen, für alle Unternehmenstypen identischen Rechtsvollzugs ebenso vermeiden lassen wie die Probleme einer willkürlichen Benachteiligung einzelner Firmen aufgrund ihrer ausländischen Herkunft. Der rechtliche Gleichbehandlungsgrundsatz wird hier, so könnte man vielleicht sagen, zum Austragungsort des Spannungsverhältnisses zwischen formaler und materialer Rationalität, und in mancher Hinsicht ist er es, der das Recht in einzelnen Feldern über sich selbst hinaustreibt und es veranlasst, sich eingehend mit der Unterschiedlichkeit der Akteure und ihren jeweiligen Handlungskapazitäten auseinanderzusetzen, gemäß dem Erfordernis, Gleiches gleich, und Ungleiches ungleich zu behandeln (vgl. Hirschberg 1981).

Auch die neuen Verantwortlichkeiten der Konzernleitung im Verhältnis zu den Tochtergesellschaften sind geeignet, Resonanzen im staatlichen Recht auszulösen, und auch hier stößt man auf Mechanismen, die es wert sind, genauer rekonstruiert zu werden - nicht zuletzt deshalb, weil sie zum Vorbild für institutionelle Anpassungen auf breiterer Front werden können. Formell sind die Tochtergesellschaften der Konzerne juristisch selbständige Unternehmen nach dortigem Recht, deren Verbindlichkeiten einzig und allein von diesen selbst zu tragen sind; haftungsrechtliche Durchgriffe auf die Muttergesellschaft sind nach gängiger Auffassung nur dort zulässig, wo diese die Entscheidungsautonomie der Tochtergesellschaft drastisch eingeengt oder die betreffende Entscheidung gegen deren Willen durchgesetzt hat. Die juristische Innovation, wie sie sich bislang freilich nur in einigen wenigen Rechtsentscheidungen ankündigt, besteht nun darin, auch die umgekehrte Konstellation, 
nämlich Fälle einer übertriebenen Steuerungsabstinenz der Zentrale als Form schuldhaften Verhaltens zu werten; im Sinne einer Vernachlässigung bestehender Sorgfalts- und Aufsichtsbefugnisse. $^{7}$

Das entsprechende Haftungskonzept lässt sich auf folgende Formel bringen Muttergesellschaften, die es versäumt haben, die verkehrsüblichen Steuerungssysteme zu installieren, oder die bei ihren internen Kontrollen gravierende Missstände aufgedeckt, diese aber nicht beseitigt haben, haften aufgrund eines Verstoßes gegen ihre Organisations- und Konzernleitungspflichten. Dass dies einen bislang nur selten genutzten und formalrechtlich nur schwach ausgeprägten Entscheidungsweg darstellt, dürfte vor allem mit der tradierten Vorstellung zusammenhängen, eine problemlose Integration der Tochtergesellschaft in den Kontext des jeweiligen Tätigkeitslandes gelinge umso besser, je weniger die Konzernleitung sich in die Vorgänge vor Ort einmische; eine Sichtweise, die durch die Vorkommnisse der jüngeren Vergangenheit als faktisch widerlegt gelten kann. Die Triebkraft für die Fortentwicklung des Rechts besteht hier im Prinzip der Verschuldenszurechnung entlang der tatsächlich bestehenden Macht- und Einflusspotentiale (»respondeat superior«; Fikentscher 1976, S. 673); ein Prinzip, das durch Prozesse der immer komplexer werdenden Arbeitsteilung zwar teilweise auf eine harte Probe gestellt wird, das gleichzeitig aber immer wieder Regelungen hervorbringt, um die Abwälzung negativer Effekte auf Dritte wirksam zu begrenzen.

Neue Anforderungen an die Rechtspraxis können sich auch aus den öffentlichen Selbstverpflichtungen der Unternehmen ergeben, die, auch wenn sie sich an ein sehr breites Publikum richten, konkrete Erwartungen bei ebenso konkreten Akteuren erzeugen, die mit dem betreffenden Unternehmen in Kontakt treten und hierbei auf die Einhaltung der Zusagen vertrauen. Unter rein formellen Aspekten wird man nur solche Willenserklärungen für justitiabel halten, die entweder in Form eines klassischen Vertrags vorliegen oder zumindest einen gewissen Rechtsbindungswillen zum Ausdruck bringen. In der Rechtspraxis finden sich demgegenüber zahlreiche Tendenzen, diese Anforderungen zu relativieren (vgl. Köndgen 1981 m.w.N.). Hier ist es das Prinzip des Vertrauensschutzes, welches das formelle Recht zu neuen Problemlösungen stimuliert, und sei es auch nur als allgemeines Unbehagen an Entscheidungen, bei denen die Nachteile eines gebrochenen Versprechens einseitig dem Empfänger aufgebürdet werden, mit der Begründung, es sei ja niemand gezwungen, den Zusagen anderer zu glauben (so aber die alte »caveat emptor «-Formel aus der frühkapitalistischen Ära, vgl. Weber $1980^{5}$, S. 384).

Wie der Überblick zeigt, sind die Anschlüsse des formalen Rechts für die emergenten Normbestände der transnationalen Sphäre äußerst vielfältig; die rechtliche Verarbeitung steckt vielfach noch in den Anfängen, und manches definiert sich bislang in erster Linie ex negativo, als Abkehr vom Paradigma der Geschlossenheit und Lückenlosigkeit des Rechts. Von einer Blindheit des Rechts in Bezug auf die Normentstehungsprozesse der (Welt-)Gesellschaft kann freilich nicht die Rede sein, im Gegenteil, nicht nur unter materialen Aspekten, auch zur Aufrechterhaltung seiner Funktionsfähigkeit und Systematik ist das Recht in vielen Fällen darauf angewiesen, die Selbstbindungstatbestände der sozialen Welt in seine Strukturen einzubeziehen.

7) Die Beispiele aus der US-amerikanischen Rechtsprechung kristallisieren sich vor allem um die sog Good Samaritan-Doktrin, der zufolge bereits die grundsätzliche Fähigkeit der Zentrale zur Einflussnahme weit reichende Pflichten der Überwachung und korrigierenden Intervention auslöst (vgl. Meier 2000, S. 496 ff). 


\section{Der Staat als aktiver Schnittstellenmanager in einer Konstellation pluraler Teil- rechtsordnungen}

In der Diskussion über die Möglichkeiten eines »Regierens ohne Weltregierung « (Zürn 1998) ist die Bedeutung der Steuerungsbeiträge Privater häufig hervorgehoben worden, die systematische Erschließung dieses Feldes steht freilich noch aus. Die Ergebnisse zu den Umweltregulativen der acht größten deutschen Chemiekonzerne sind ein möglicher Schritt in diese Richtung; wichtige weitere Anwendungsfälle wären Chemiekonzerne von geringerer Größe und/ oder aus anderen Herkunftsländern, Unternehmen aus dem Bergbau- und Erdölsektor, der Automobil-, der Elektro- und der Maschinenbaubranche, des Einzelhandels, des Lebensmittelsektors und der Textilbranche (zu letzteren vgl. Bair/ Gereffi 2000). Die emergenten Steuerungsarrangements der Wirtschaftswelt füllen Räume, die von staatlicher Herrschaft bislang nur schwach durchdrungen sind, was freilich nicht bedeutet, dass diese Räume dauerhaft gegen staatliche Zugriffe abgeriegelt würden. Aufgrund der hohen Praxisrelevanz der informellen Mechanismen, ihrer erwartungs- und strukturbildenden Funktion, kommt es zu zahlreichen Wechselwirkungen mit dem geltenden staatlichen Recht, das von vielen Seiten her mit den Geltungsansprüchen des transnationalen Rechts konfrontiert wird und hierdurch selbst, zumindest in Ansätzen, seine Identität verändert.

In mancher Hinsicht zeichnet sich in diesen Prozessen eine Transformation des territorial gebundenen Nationalstaats hin zu einem globalen »Interdependenz- und Schnittstellenmanagers « ab (Messner 2002, S. 28). Die Behörden und Gerichte der verschiedenen Länder werden zunehmend in die Steuerungsprozesse der transnationalen Sphäre eingeflochten, und in vielen Fällen sehen sie sich dazu veranlasst, ihre Ermittlungs- und Steuerungsaktivitäten über die nationalstaatlich definierten Grenzen hinaus auszudehnen. Die Tätigkeitsländer der Konzerne können deren selbst gesetztes Recht aufgreifen und hierdurch zu Anforderungen gelangen, die ohne die Kenntnis dieser Standards nicht möglich gewesen wären. Auch die staatlichen Institutionen in den Herkunftsländern sehen sich vermehrt mit Geltungsansprüchen extraterritorialen Ursprungs konfrontiert, darunter den Beschwerden und Klagen von Geschädigten vor Ort, und sie können diese Ansprüche nicht mehr ohne weiteres mit dem Argument abwehren, bei den Tochtergesellschaften handle es sich um juristisch selbständige Einheiten, die dem Einfluss der Muttergesellschaften schon per definitionem entzogen seien.

Globalisierung sollte daher nicht vorschnell mit Entstaatlichung gleichgesetzt werden; wo es gelingt, die nationalen Institutionen adäquat an die neuen Herausforderungen anzupassen, bleibt der Staat ein wichtiger Akteur im globalen Steuerungsprozess. Zum Teil agiert er als wichtiger Initiator von Selbstregulierung, zum Teil trägt er durch flankierende Maßnahmen dazu bei, die Zuverlässigkeit der privaten Regulative zu steigern, und in vielen Fällen repräsentiert er eine wichtige Anlaufstation für Ansprüche und Forderungen, die immer dann entstehen, wenn Selbstregulierung versagt oder an ihre Grenzen gelangt. Prinzipiell trifft auch hier zu, was über die verschiedenen Formen indirekter und >reflexiver Steuerung im innerstaatlichen Verhältnis gesagt wird: bei seiner Suche nach sinnvollen Steuerungsmechanismen muss der Staat intensiv auf die Intelligenz und die Problemnähe gesellschaftlicher Akteure zurückgreifen (vgl. Mayntz/Scharpf 1995). Der empirischen Sozialforschung kommt hierbei eine wichtige Brücken- und Aufklärungsfunktion zu, gilt es doch, diese Intelligenz, um sie zu nutzen, zunächst einmal analytisch zu durchdringen.

\section{Literatur}

Austin, John 2002: Zur Theorie der Sprechakte (How to do Things with Words). Stuttgart.

Bair, Jennifer/ Gereffi, Gary 2000: Industrielle Entwicklung, Netzwerke und Beschäftigung in der Bekleidungsindustrie. In: Ch. Dörrenbächer/ D. Plewe (Hg.), Grenzenlose Kontrolle? Organisatorischer Wandel und politische Macht multinationaler Unternehmen. Berlin, S. $195 \mathrm{ff}$. 
Bartlett, Christopher/ Goshal, Sumantra 1999: Managing across Borders - the Transnational Solution. Boston.

Beck, Ulrich 1997: Was ist Globalisierung? Irrtümer des Globalismus - Antworten auf Globalisierung. Frankfurt/ Main.

Ders. 2002: Macht und Gegenmacht im globalen Zeitalter. Neue weltpolitische Ökonomie. Frankfurt/ Main.

Böhm, Ursula 2000: Umweltorientiertes Management multinationaler Unternehmen in der VR China. Baden-Baden.

Böttger, Katja 2002: Die Umweltpflichtigkeit von Auslandsdirektinvestitionen im Völkerrecht. BadenBaden.

Bohnsack, Ralf 1993: Rekonstruktive Sozialforschung. Einführung in Methodologie und Praxis qualitativer Forschung. Opladen.

Bovens, Mark 1998: The Quest for Responsibility. Accountability and Citizenship in Complex Organizations. Cambridge.

Chandler, Alfred 1966: Strategy and Structure, Cambridge u. London.

Cicourel, Aaron 1973: Basisregeln und normative Regeln im Prozess des Aushandelns von Status und Rolle. In: Arbeitsgruppe Bielefelder Soziologen. Alltagswissen, Interaktion und gesellschaftliche Wirklichkeit. Bd. I, S. $147 \mathrm{ff}$.

Cutler, Claire 2002: Private International Regimes and Interfirm Cooperation. In: Hall/ Biersteker 2002 (a.a.O.), S. 23-42.

Desai, Uday 1998 (Hg.): Ecological Policy and Politics in Developing Countries. New York.

Ehrlich, Eugen 1989: Grundlegung der Soziologie des Rechts. Berlin.

Fikentscher, Wolfgang 1976: Schuldrecht. Berlin / New York.

Fuchs, Peter 1997: Transnationale Konzerne als Akteure 'nachhaltiger' Entwicklung? In: Nord-Süd aktuell, S. $284 \mathrm{ff}$.

Gündling, Lothar 1998: Umweltrechtsberatung und Institutionenentwicklung im Umweltbereich. Wiesbaden.

Günter, Klaus/ Randeria, Shalini 2001: Recht, Kultur und Gesellschaft im Prozess der Globalisierung. Werner Reimers Stiftung.

Hall, Rodney/ Biersteker, Thomas (Hg.) 2002: The Emergence of Private Authority in Global Governance. Cambridge.

Herberg, Martin 2001: Codes of Conduct und kommunikative Vernunft. Rechtssoziologische Überlegungen zu den Umweltleitlinien transnationaler Chemiekonzerne, in ZfR (22), S. 25 ff.

Ders. 2005: Erzeugen multinationale Unternehmen ihr eigenes Umweltrecht? In: G. Winter, Die Umweltverantwortung multinationaler Unternehmen, Baden-Baden, S. 73.

Hirschberg, Lothar 1981: Der Grundsatz der Verhältnismäßigkeit. Göttingen.

Köndgen, Johannes 1981: Selbstbindung ohne Vertrag. Zur Haftung aus geschäftsbezogenem Handeln. Tübingen.

Lampe, Ernst-Joachim (Hg.) 1995: Rechtsgleichheit und Rechtspluralismus. Baden-Baden.

Leonard, Jeffrey 1988: Pollution and the Struggle for the World Product: Multinational Corporations, Environment and International Comparative Advantage. Cambridge.

Luhmann, Niklas 1987: Rechtssoziologie. Opladen.

Mannheim, Karl 1980: Strukturen des Denkens. Frankf./ Main.

Mayntz, Renatel Scharpf, Fritz 1995: Gesellschaftliche Selbstregulierung und politische Steuerung. Frankf./ Main.

Meier, Werner 2000: Grenzüberschreitender Durchgriff in der Unternehmensgruppe nach US-amerikanischem Recht. Frankf./ Main.

Messner, Dirk 2002: Nationalstaaten in der Global Governance-Architektur. Duisburg, INEF-Report. 
Neff, Alan 1990: Not in Their Backyards Either. A Proposal for a Foreign Environmental Practices Act, Ecology Law Quarterly (17), S. $477 \mathrm{ff.}$

Oevermann, Ulrich 1983: Zur Sache. Die Bedeutung von Adornos methodologischem Selbstverständnis für die Begründung einer materialen soziologischen Strukturanalyse. In: J. Habermas/ L. v. Friedeburg (Hg.): Adorno-Konferenz, Frankf./ Main, S. 234 ff.

Oevermann, Ulrich/Schuster, Leo/Simm, Andreas 1985: Zum Problem der Perseveranz in Delikttyp und modus operandi. Wiesbaden, BKA-Reihe.

Ott, Walter 1992: Der Rechtspositivismus. Kritische Würdigung auf der Grundlage eines juristischen Pragmatismus. Berlin.

Pauly, Louis W. 2002: Global Finance, Political Authority, and the Problem of Legitimation. In: Hall/ Biersteker 2002 (a.a.O.), S. 76-90.

Robertson, Roland 1998: Glokalisierung: Homogenität und Heterogenität in Raum und Zeit. In: U. Beck (Hg.), Perspektiven der Weltgesellschaft. Frankf./ Main, S. 192 ff.

Rolf, Eckard 1997: Illokutionäre Kräfte. Grundbegriffe der Illokutionslogik. Opladen.

Rosenau, James 1992: Governance, Order and Change in World Politics. In: Ders./ E. O. Czempiel (Hg.), Governance without Government. Order and Change in World Politics. Cambridge, S. 3-17.

Santos, Boaventura de Sousa 1995: Toward a New Common Sense. Law, Science and Politics in the Paradigmatic Transition. New York u. London.

Scherer, Andreas 2003: Multinationale Unternehmen und Globalisierung. Zur Neuorientierung der Theorie der multinationalen Unternehmung. Heidelberg.

Schmidt-Preuß, Matthias 1998: Verwaltung und Verwaltungsrecht zwischen gesellschaftlicher Selbstregulierung und staatlicher Steuerung. VVdStRL (56), S. $160 \mathrm{ff}$.

Stöckl, Walter 1996: Ökologieorientierte Führung multinationaler Unternehmen in Entwicklungsländern. Wiesbaden.

Teubner, Gunther 1991: Unitas Multiplex - Das Konzernrecht in der neuen Dezentralität der Unternehmensgruppe. In: Zeitschrift für Unternehmens- und Gesellschaftsrecht 20 (2), S. 189 ff.

Ders. 2000: Des Königs viele Leiber. Die Selbstdekonstruktion der Hierarchie des Rechts. In: H. Brunkhorst/ M. Kettner (Hg.), Globalisierung und Demokratie. Frankfurt/ Main S. 240-273.

Weber, Max 19805: Wirtschaft und Gesellschaft. Grundriss der verstehenden Soziologie. Tübingen, Mohr.

Weidner, Helmut/Jänicke, Martin 2001: Die umweltpolitischen Chancen der Globalisierung. In: L. Röller/ Ch. Wey (Hg.), Die soziale Marktwirtschaft in der neuen Weltwirtschaft. WZB-Jahrbuch, Berlin, S. $293 \mathrm{ff}$.

Zürn, Michael 1998: Regieren jenseits des Nationalstaats. Globalisierung und Denationalisierung als Chance. Frankf./ Main.

Martin Herberg

SFB $597 »$ Staatlichkeit im Wandel«

Universität Bremen, Linzerstraße 9a, 28334 Bremen martin.herberg@sfb597.uni-bremen.de 